\title{
Novel Performance Evaluation of Thermal Camera Based on VOx Bolometer Focal Plane Array via Analysis of Sigma NETD, Mean NETD, and Roughness Index
}

\author{
Cheng-De Lee, ${ }^{1}$ Shiang-Feng Tang, ${ }^{2 *}$ and Tzu -Chiang Chen $^{3}$ \\ ${ }^{1}$ School of Defense Science, Chung Cheng Institute of Technology, National Defense University, \\ No. 75, Shiyuan Rd., Daxi Dist., Taoyuan City 335, Taiwan \\ ${ }^{2}$ Materials \& Electro-Optics Division, National Chung-Shan Institute Science of Technology, \\ No. 615, Shiyuan Rd., Longtan Dist., Taoyuan City 325, Taiwan \\ ${ }^{3}$ Department of Electrical and Electronic Engineering, Chung Cheng Institute of Technology, \\ National Defense University, No. 75, Shiyuan Rd., Daxi Dist., Taoyuan City 335, Taiwan
}

(Received January 22, 2018; accepted April 2, 2018)

Keywords: roughness index (RI), noise equivalent temperature difference (NETD), full width at half maximum (FWHM), non-uniformity correction (NUC)

With recent advancements in thermal imaging, the evaluation of thermal imaging performance has become important. In this study, the thermal-camera performance parameters of roughness index $(R I)$, noise equivalent temperature difference (NETD), and the full width at half maximum (FWHM) of a statistical NETD histogram are investigated and compared by varying the integration times at different operating temperatures for vanadium oxide (VOx)based microbolometer focal plane arrays (FPAs) with the use of the Matlab algorithm platform. The quantitative performance assessment of an uncooled VOx microbolometer-based thermal imager, which was designed and fabricated by researchers from the National Chung-Shan Institute Science of Technology (NCSIST), Taiwan, and the National Optics Institute (INO), Canada, is proposed systematically. Explicitly, the uncompressed video data streams before non-uniformity correction (NUC) using two-point temperature calibration were acquired for integration times of $16.67,33.33$, and $50 \mathrm{~ms}$ at three operating temperatures of 10,15 , and 20 ${ }^{\circ} \mathrm{C}$. The results from the estimations of NETD, FWHM of the NETD histogram, and the RI for the thermal imager are discussed for the imaging performance evaluation in different infrared operation scenarios. We believe that our findings can significantly contribute to the further development of IR imaging technology.

\section{Introduction}

Infrared (IR) imaging is based on the observation that there is extremely low absorption of IR irradiation in the IR atmospheric window; consequently, the IR wavelength regions between 3 to $5 \mu \mathrm{m}$ and 8 to $14 \mu \mathrm{m}$ are employed for imaging purposes. In this context, the past few decades have witnessed rapid developments in the field of IR detector technology, particularly

*Corresponding author: e-mail: shiangfengtang@gmail.com http://dx.doi.org/10.18494/SAM.2018.1913 
for military applications. On the other hand, commercial thermal imagers have recently become the subject of scientific research and industrial application. Nowadays, lightweight and function-effective thermal imagers enable firefighters to easily see through smoke in their search for victims and to ensure that flames are fully extinguished. Other niche applications involving IR detection include security, night vision for driving, non-contact temperature measurement, defect inspection, and medical imaging applications. ${ }^{(1,2)}$

A commonly used figure of merit for IR detectors is the noise equivalent temperature difference $(N E T D) .{ }^{(3-7)}$ In addition, the parameter of roughness index $(R I)$ has also been employed recently; ${ }^{(8-10)}$ this parameter represents the estimation of the spatial and time-variant noise for an IR image, and it represents the difference between each pixel of every corrected image and its corresponding left neighbor. This difference arises from imperfections in the fabrication of the focal plane array (FPA) and the readout circuitry. In this study, we evaluated the performance of a thermal camera based on these parameters.

This paper is structured as follows: the experimental setup, NETD, full width at half maximum $(F W H M)$ of the NETD, and RI are described in Sect. 2. Section 3 presents our experimental results, followed by a quantitative performance comparison of the imager for different operating scenarios. Finally, conclusions are provided in Sect. 4.

\section{Setup for Performance Estimation of Two-dimensional Infrared Focal Plane Array}

Figure 1 shows the schematic and configuration diagram of the experimental setup used in our study for the acquisition and handling of uncompressed video data streams, which are evaluated with the RI proposed by Moreno et al. ${ }^{(10)}$ along with NETD and FWHM of the
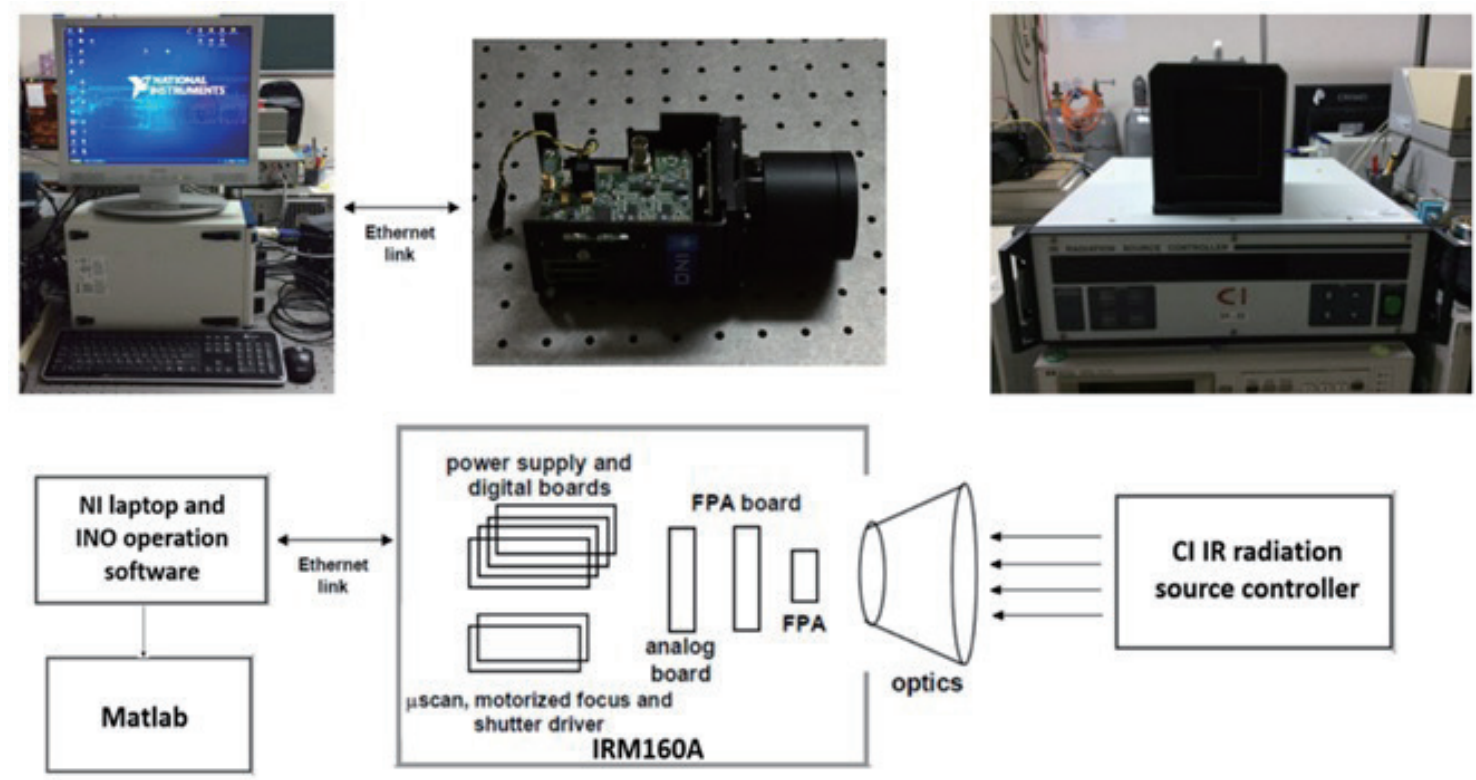

Fig. 1. (Color online) Experimental setup of IRXCAM-160A configuration and its schematic for the microbolometer-focal-plane-array-mounted IRXCAM-160A. 
NETD histogram using the commercial Matlab algorithm platform. The IR imaging evaluation system used in our experimental setup is the INO IRXCAM-160A camera, which is mounted with an uncooled microbolometer FPA with a size of $160 \times 120$ pixels and pitch of $52 \mu \mathrm{m}$. The digital data stream output from the camera is transmitted to a laptop via a Gigabit Ethernet connection. The maximum frame rate in the full $160 \times 120$ pixels format is $30 \mathrm{~Hz}$. The NETD is calculated with the optics of F/0.86 at an ambient temperature of $300 \mathrm{~K}$. To achieve a highuniformity image, two-point correction is carried out on the image using a flat-shaped CI blackbody. The IR image is obtained with a given microbolometer FPA mounted at the focal position with the optic lens (JANOS Technology Surnia Lenses 40588-AA1). A microshutter is assembled to provide a real-time offset update on the IR image. A National Instrument acquisition platform (NI PXIe-8105 embedded controller in PXI-1042Q express chassis) is used to communicate with the IRXCAM-160A camera, to setup initial operation conditions, and to process the raw streaming image signal and data (offset and gain correction and pixel replacement for image improvement). Finally, the post-processed image is evaluated. ${ }^{(11)}$ The irradiated IR energy captured by the current-biased microbolometer FPA changes the resistance of the vanadium oxide detector, thus generating a proportional voltage signal that is used in turn to generate a gray-level intensity value via an external A/D converter chip for each row or column. Meanwhile, the streaming data of the FPA for 30, 15, and $10 \mathrm{frames} / \mathrm{s}$ (fps) is extracted at a sample rate of 16 bits per pixel. The resulting digital images are saved in the RAW image format, which contains the full resolution data as read out from the camera's image sensors, to the NI PXI platform via the Gigabit Ethernet link between the IRXCAM-160A camera and the NI PXI acquisition platform. The streaming data corresponding to 300 frames for each pixel is obtained to calculate the distribution of the NETD histogram and RI using Matlab software. Figure 2 shows the flowchart for calculating the statistical NETD histogram and RI under

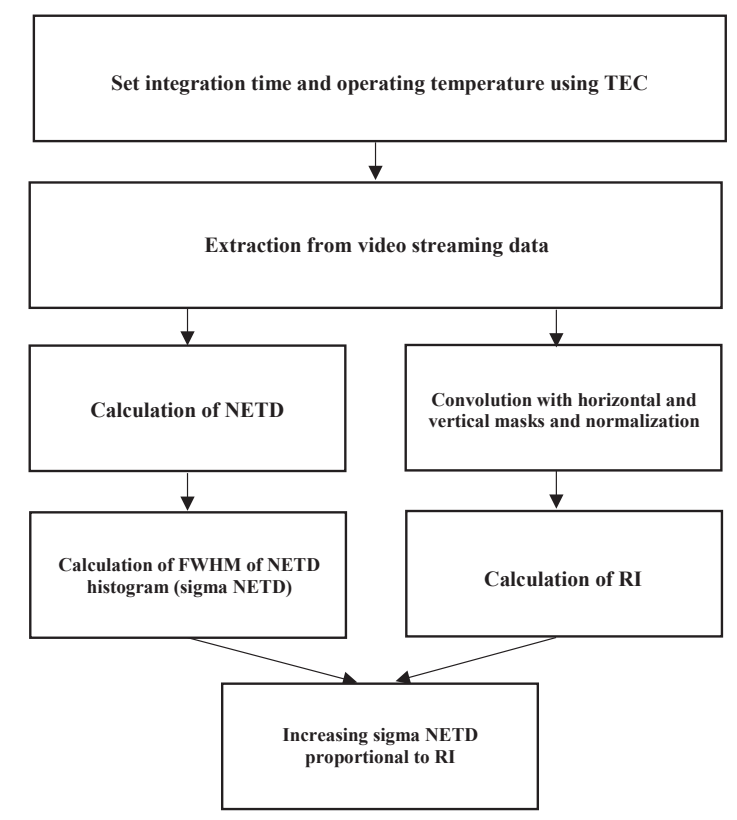

Fig. 2. Flowchart of performance evaluation using FWHM of NETD histogram and roughness index. 
specific FPA operating conditions. The correlation between these two parameters is obtained to systematically perform a comprehensive analysis. The specifications of the microbolometer FPA, IR optics, and experimental setup with the INO IRXCAM-160A are listed in Table 1. Following previous studies, ${ }^{(3,10)}$ the NETD histogram incorporated with its FWHM (sigma $N E T D)$ and $R I$ are introduced as evaluation parameters. The quantitative parameters of the NETD histogram and $R I$ represent the FPA

In this section, we present a concise description of the individual components in the acquisition system, and we define the main requirements for the performance computation. The details of the processes of computation and the parameters described are defined in the following sub-sections.

\subsection{Noise equivalent temperature difference}

The evaluation of the time-variant noise results in a three-dimensional signal intensity data set in the FPA under specific operating conditions. The time-variant noise is calculated by averaging the standard deviation of each pixel output over the duration of 30 frames of a uniform temperature calibration scene. In addition to the two spatial dimensions of the rows and columns in the detector array, the signal transient along consecutive frames and the responsivity as a function of various uniform background irradiation temperatures are recorded. The temperature resolution of IR FPA is limited by time-variant and spatial noise. In general, the spatial noise is partially removed using corrective procedures. These corrective procedures reduce the spatial noise to a magnitude below the time-variant noise (i.e., the temporal noise). ${ }^{(12)}$

The time-variant noise $\sigma_{t n}$ of the IR FPA is defined by the root mean square (RMS) value of the temporal noise of the individual pixels. The time-variant noise $\sigma_{t n, i}$ of an individual pixel $i$ in frame $j$ is calculated using the following relation:

$$
\sigma_{t n, i}=\sqrt{\frac{\sum_{j=1}^{N}\left(x_{i, j}-<x_{i}>\right)^{2}}{N-1}},
$$

Table 1

Specifications of INO IRXCAM-160A.

\begin{tabular}{lc}
\hline Specifications of microbolometer FPA, IR optics, and experimental setup with INO IRXCAM-160A \\
\hline Detector specification, FPA format, and IR window & $\begin{array}{c}\text { Uncooled VOx microbolometer FPA } \\
160 \times 120 \text { pixels, 52- } \mu \mathrm{m} \text { pitch } \\
\text { Germanium }\end{array}$ \\
\hline Camera module & INO IRXCAM \\
\hline Optics & JANOS Technology Surnia Lenses 40588- \\
AA1/F\#0.86
\end{tabular}


where $N$ represents the number of frames recorded at a specific uniform irradiation of the blackbody temperature $T_{B B}$, and $x_{i, j}$ is the signal amplitude of pixel $i$ in frame $j$. The mean value $<x_{i}>$ is defined as

$$
<x_{i}>=\frac{\sum_{j=1}^{N} x_{i, j}}{N}
$$

The mean time-variant noise $\sigma_{t n}$ of the total detector array consisting of $n$ individual pixels is calculated as

$$
\sigma_{t n}=\sqrt{\frac{\sum_{i=1}^{N} \sigma_{t n, i}^{2}}{n}} .
$$

The time-variant noise $\sigma_{t n}$ is related to the background temperature $T_{B B}$ and to the integration time of the detector array and the method of calculating the standard deviation results in an optimistic result, as $1 / f$ noise is partially removed from the data in the equation. An improvement in the temperature resolution is obtained by increasing the background temperature and the integration time of the FPA. The unified response curve $<y\left(T_{B B}\right)>$ to which all pixels are corrected is defined by the array signal average as

$$
y\left(T_{B B}\right)=\frac{\sum_{i=1}^{n}<x_{i}\left(T_{B B}\right)>}{n} .
$$

The derivative of $<y\left(T_{B B}\right)>$ with respect to $T_{B B}$ yields the responsivity required to convert the time-variant noise $\sigma_{t n}$ into the NETD, ${ }^{(3,7,12)}$ the conversion of which is achieved with the signal transform function (SiTF).

$$
\operatorname{NETD}\left(T_{B B}\right)=\sigma_{t n}\left(T_{B B}\right) \frac{d<y\left(T_{B B}\right)>}{d T_{B B}}
$$

For typical data sets as they are recorded in this analysis, Eq. (5) is used to determine the NETD. Moreover, sigma NETD is defined as the FWHM of the extent of the NETD histogram.

\section{$2.2 R I$}

The dimensionless figure of merit $R I$ is defined for any frame of the video stream as ${ }^{(8-10)}$

$$
R I=\frac{\left\|h * x_{i, j}\right\|_{1}+\left\|v * x_{i, j}\right\|_{1}}{\left\|x_{i, j}\right\|_{1}} .
$$


Here, $h$ represents the horizontal mask or filter $[1,-1]$, the vertical filter $[1,-1]^{\mathrm{T}}$, $\left\|x_{i, j}\right\|_{1}$ the $L_{1}$ norm of $x_{i, j}$, and $*$ the discrete convolution operator. In the denominator of Eq. (6), normalization by $\left\|x_{i, j}\right\|_{1}$ makes $R I$ invariant to the grey scale and image dimensions under scaling. The two terms in the numerator of Eq. (6), $\left\|h * x_{i, j}\right\|_{1}$ and $\left\|v * x_{i, j}\right\|_{1}$, measure the variations between neighboring pixels along the horizontal and vertical directions of the image, respectively. It is to be noted that $R I$ is zero for a uniform image, and it increases with the pixelto-pixel response variations in arrays and sensors of the imager under temporal noise free. As non-uniformity is used to describe the FPA, this characteristic is relevant to the examination of bare FPAs, because it can be used to determine the level of compensation required to satisfy the specifications of sensor performance. Moreover, RI can be also used as a measure of nonuniformity correction and a useful indicator of the thermal imaging performance affected by the presence of FPA noise.

Table 2 summarizes the resource utilizations in terms of computation time. Concerning the utilization of CPU time, the calculation of the $R I$ is more cost-effective than that of the NETD histogram with its FWHM. The computation time for the RI estimation is reduced by about $50 \%$ with respect to that for the NETD histogram and the FWHM of the statistical NETD. Nevertheless, the FWHM of the NETD histogram forms an important statistical physics concept.

\section{Results and Discussions}

To examine the performance of the VOx microbolometer FPA under varying integration times at three different operating temperatures as achieved via the adjustment of a thermoelectric cooler (TEC), the statistical NETD histogram is calculated from 30 sequential frames, and $R I$ is calculated from each 50 -frame interval of the 300 -frame digital video streaming data captured by the experimental setup. The electrical bandwidth is determined by the integration time of the microbolometer, and this bandwidth is significant for analyzing thermal fluctuation noise and suitably setting the integration time.

Figure 3 shows the dependence of the NETD on the integration times at three different operating temperatures. It is obvious that the mean NETD values decrease while the integration times increase at the three different operating temperatures of 10,15 , and $20{ }^{\circ} \mathrm{C}$. The lower operating temperature of the VOx microbolometer FPA, the smaller the NETD. The reduction in the thermal noise of the thermistor-type bolometer detector with decreasing operating temperature can easily be deduced. However, the integration times are inversely proportional to the NETD, probably due to the increase in detector sensitivity and signal dynamic value. ${ }^{(13)}$

Figure 4 depicts the evaluation results for the NETD histogram and its FWHM for integration times of $16.67,33.33$, and $50 \mathrm{~ms}$ at operating temperatures of 20,15 , and $10{ }^{\circ} \mathrm{C}$ for the

Table 2

Computation times required for calculation of the three parameters.

\begin{tabular}{lccc}
\hline $\begin{array}{l}\text { Time expense for } \\
\text { parameter computation }\end{array}$ & NETD histogram & $\begin{array}{c}\text { FWHM of NETD histogram } \\
\text { (sigma NETD) }\end{array}$ & RI \\
\hline Time (s) & 2.821 & 1.013 & 1.897 \\
\hline
\end{tabular}




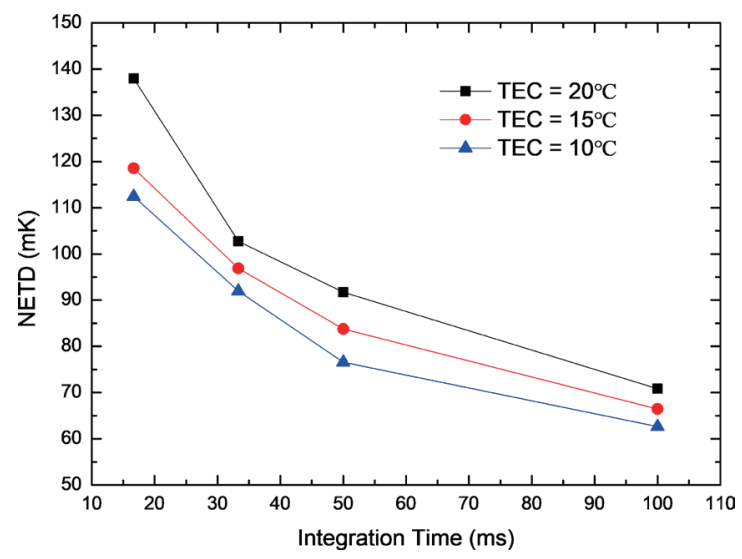

Fig. 3. (Color online) Mean NETD of microbolometer FPA as a function of integration time at three different operating temperatures.

IRXCam160 camera mounted with the IR optics F/0.86 lens. After calculations and analyses, under a nominal operating temperature of $20{ }^{\circ} \mathrm{C}$, the average NETDs are obtained as 138 , 103, and $92 \mathrm{mK}$ for integration times of $16.67,33.33$, and $50 \mathrm{~ms}$, respectively. Furthermore, when the operating temperature is as low as $10{ }^{\circ} \mathrm{C}$, the mean NETD decreases to $\sim 77 \mathrm{mK}$ for an integration time of $50 \mathrm{~ms}$. The sigma NETD (FWHM of the NETD histogram) value is also the lowest [i.e., below $30 \mathrm{mK}$, Fig. 4(i)] for these conditions. This result can be mainly attributed to the contribution of different types of noise (Johnson noise, thermal fluctuation noise, and background scene noise) to the NETD histogram. ${ }^{(13)}$ The major noise source is the Johnson noise related to the bandwidth of the bolometer FPA. But the electrical bandwidth is determined by the integration time of the bias pulse to measure the resistance of the bolometer, and this bandwidth is important for analyzing the system contributions of Johnson noise. The electrical biasing generates Joule heating and causes a change in the resistance of the bolometer detector. If the bias current is too high or biased for a long duration, it results in excessive heating and permanent damage to the bolometer detector. Similarly, if the bias current is too low, it results in low responsivity in the bolometer detector. If the bolometer detector is biased for a long duration, it results in high temperature drift in the bolometer detector due to selfheating. ${ }^{(14)}$ Accordingly, for our experimental setup, the bolometers are pulse biased with a nominal value of bias current. The second source of noise stems from temperature fluctuations in the detector caused by heat conductance variations between the bolometer and the surrounding substrate with which the bolometer pixel is in thermal contact. ${ }^{(15)}$ A third source is the background scene noise resulting from irradiative heat exchange between the bolometer and the surrounding environment that is being observed. An increase in integration time related to the bandwidth results in increase in the bolometer response while the noise figure is identical under the same operating temperature; consequently, the NETD decreases. Moreover, sigma NETD decreases with increasing integration times, and it increases with increases in the imaging frame rate and operating temperature. This may be to due to the larger deviation of each pixel driven by a higher image frame rate and operating temperature. 


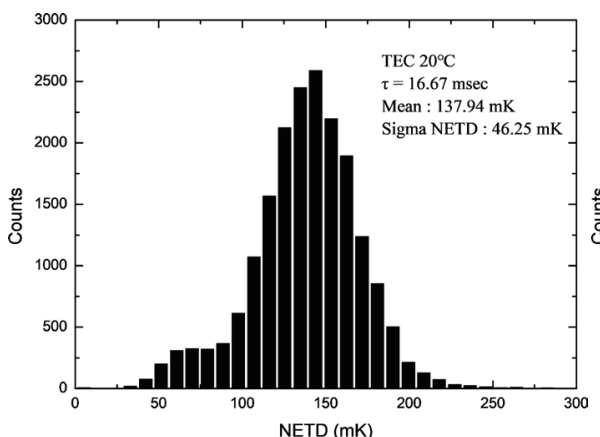

(a)

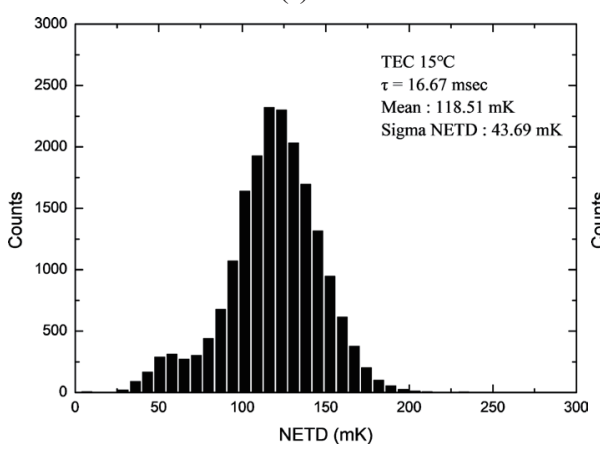

(d)

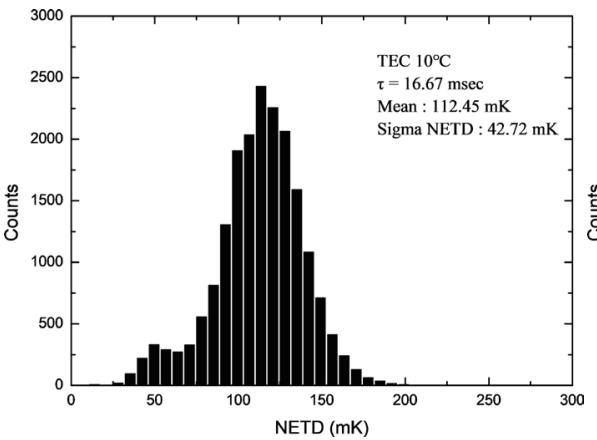

(g)

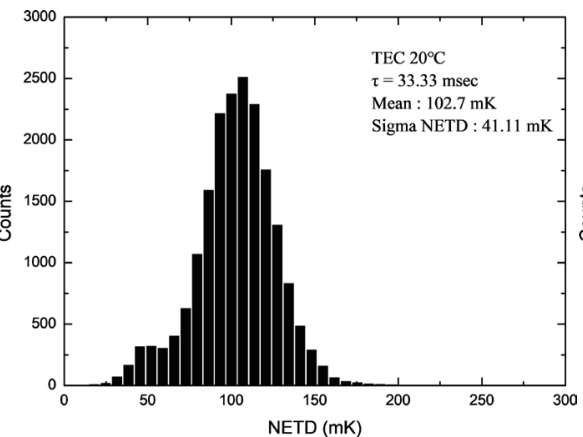

(b)

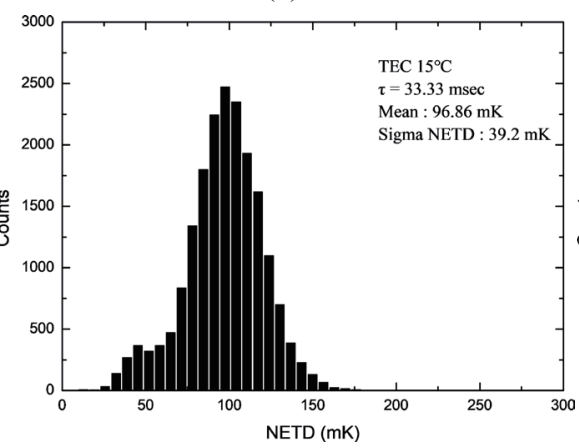

(e)

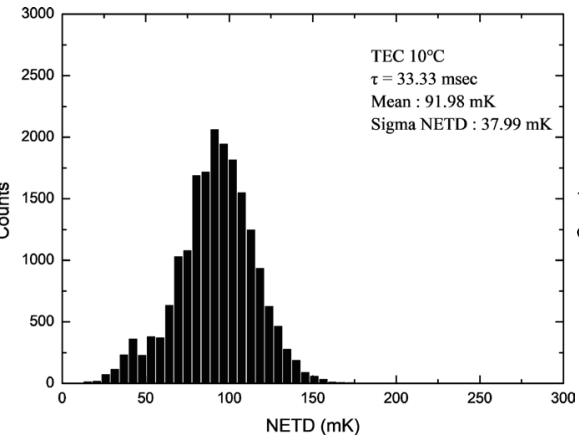

(h)

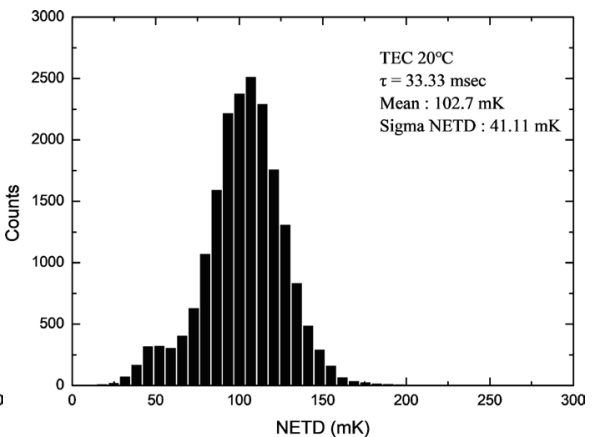

(c)

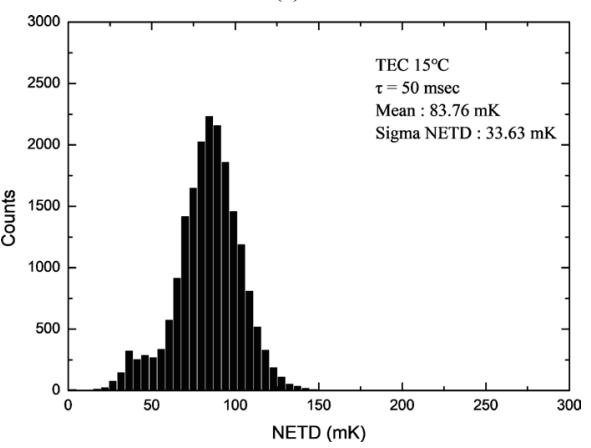

(f)

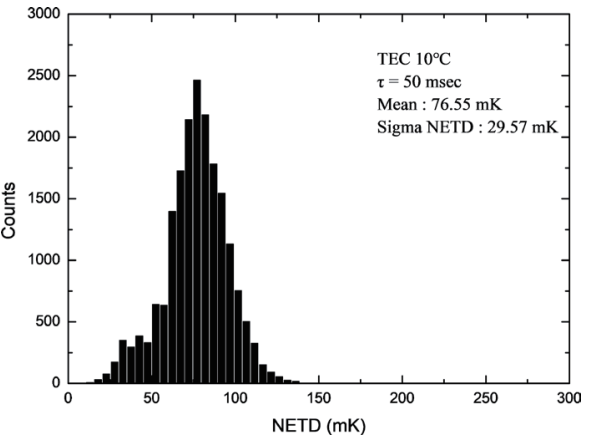

(i)

Fig. 4. Comparison of statistical NETD histograms. (a)-(c) TEC temperature set-point (TEC $\left.T_{s p}\right)=20{ }^{\circ} \mathrm{C}, \tau=$ 16.67, 33.33, and $50 \mathrm{~ms}$, (d)-(f) TEC $T_{s p}=15^{\circ} \mathrm{C}, \tau=16.67,33.33$, and $50 \mathrm{~ms}$, and (g)-(i) TEC $T_{s p}=10^{\circ} \mathrm{C}, \tau=16.67$, 33.33 , and $50 \mathrm{~ms}$.

From Eq. (6), the normalized $R I$ values that depend on the integration times and operating temperature are calculated and listed in Table 3. These values are compared with the ratio of sigma NETD and integration time normalized by scaling between 0 and 1 (Fig. 5). An identical approach is adopted for the normalized curve under a nominal operating temperature of $20{ }^{\circ} \mathrm{C}$. We can obviously conclude that the $R I$ and the normalized sigma NETD/integration time by the scaling of $[0,1]$ can both be regarded as the correlation indexes for the evaluation of FPA uniformity and noise figure depending on operating temperatures and integration times shown in Fig. 5.

Initially, the continuous video streaming data extracted by the NI PXI acquisition card with an interval cycle of 300 frames was not used for recalibration in the duty cycling period. Then, 
Table 3

Integration times, sigma $N E T D$, sigma $N E T D / \tau$, mean $N E T D$, and $R I$ for operating temperatures of 20, 15, and 10 ${ }^{\circ} \mathrm{C}$.

\begin{tabular}{lccccc}
\hline $\begin{array}{l}\text { TEC, } T_{s p} \\
\left({ }^{\circ} \mathrm{C}\right)\end{array}$ & $\begin{array}{c}\text { Integration Time } \tau \\
(\mathrm{ms})\end{array}$ & $\begin{array}{c}\text { Sigma NETD } \\
(\mathrm{mK})\end{array}$ & $\begin{array}{c}\text { Sigma } N E T D / \tau \\
(\mathrm{K} / \mathrm{s})\end{array}$ & $\begin{array}{c}\text { Mean NETD } \\
(\mathrm{mK})\end{array}$ & $\begin{array}{c}\text { RI } \\
\left(\times 10^{-3}\right)\end{array}$ \\
\hline \multirow{3}{*}{20} & 16.67 & 46.3 & 2.77 & 137.9 & 19.4 \\
& 33.33 & 41.1 & 1.23 & 102.7 & 16.1 \\
& 50 & 36.9 & 0.74 & 91.7 & 14.8 \\
\hline \multirow{3}{*}{15} & 16.67 & 43.7 & 2.62 & 118.5 & 17.3 \\
& 33.33 & 39.2 & 1.18 & 96.9 & 15.6 \\
& 50 & 33.6 & 0.67 & 83.8 & 14.2 \\
\hline \multirow{3}{*}{10} & 16.67 & 42.7 & 2.56 & 112.5 & 16.5 \\
& 33.33 & 38.0 & 1.14 & 92.0 & 13.8 \\
& 50 & 29.6 & 0.59 & 76.6 & 11.8 \\
\hline
\end{tabular}

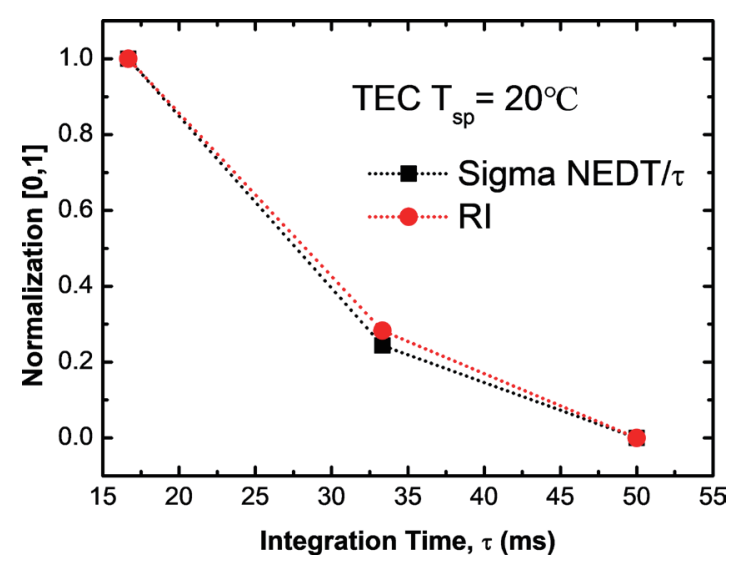

Fig. 5. (Color online) Comparison of the normalized sigma $N E T D / \tau$ with $R I$ for TEC $T_{s p}=20^{\circ} \mathrm{C}$ as function of $\tau$ ranging from 16.67 to $50 \mathrm{~ms}$.

to achieve high uniformity in a thermal image, the thermal camera must be calibrated using the two-point temperature non-uniformity correction scheme. Figure 6 shows the $R I$ of the VOxbolometer-based FPA for varying integration times $\tau$ at the three operating temperatures (20, 15 , and $10^{\circ} \mathrm{C}$ ) as a function of the image number frame as computed in the Matlab platform using high double-precision format computation data. The IRXCAM-160A thermal camera is fundamentally limited by temperature fluctuation noise arising from radiant power exchange with the background scene, and it is usually favored in a longer wavelength spectral range than the IR camera based on photon detectors, which are essentially limited by generationrecombination noise arising from photon exchange with the radiation background. Each active bolometer pixel on the FPA is sensitive to IR irradiation and thermally shorted to the silicon substrate, and therefore, there is a change in the resistance due to the pulsed constant bias applied per active bolometer pixel related to the underlying integration capacitor of the readout integrated circuit (ROIC) during a given interval of integration time. The integration time increases and the readout voltage adds up continuously so that the output signal increases linearly in contrast to the constant noise due to resistance fluctuations on the bolometer pixel 


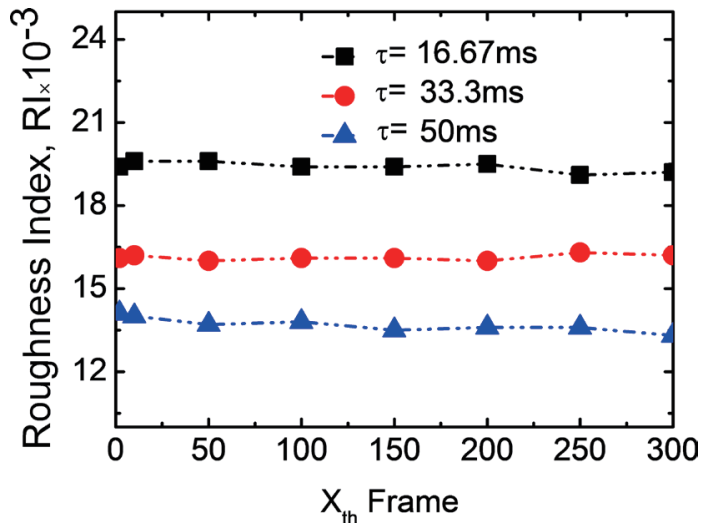

(a)

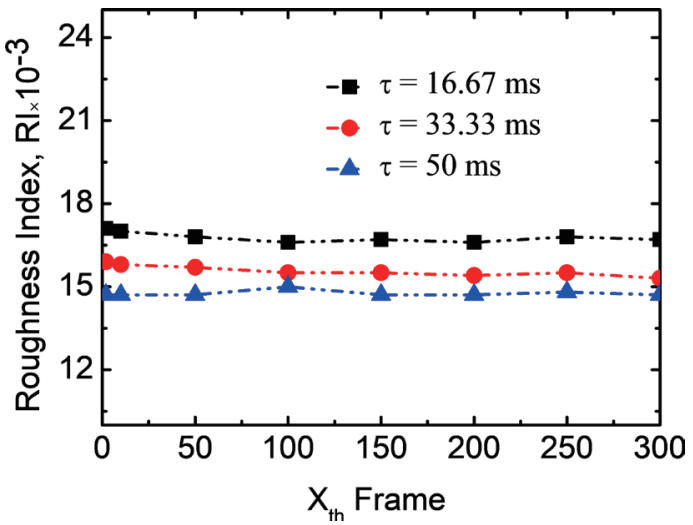

(b)

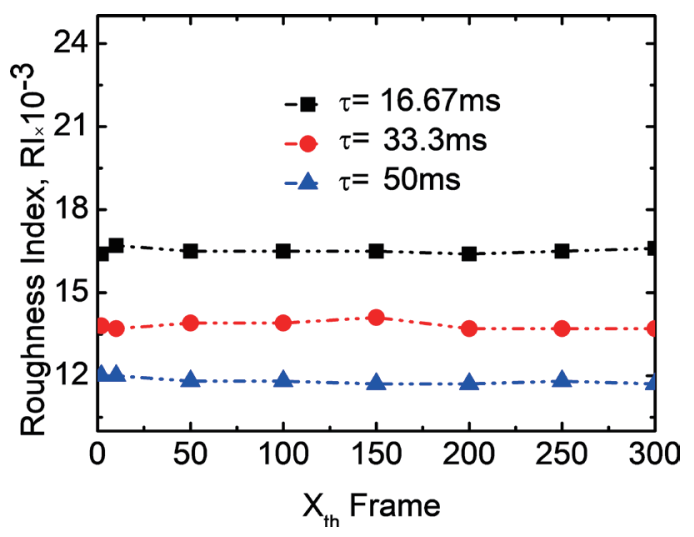

(c)

Fig. 6. (Color online) $R I$ as a function of the image number frame as acquired by the NI-PXI grabber and the FPA irradiated by the flat-shaped CI blackbody at $30{ }^{\circ} \mathrm{C}$ for different integration times $\tau=16.67,33.3$, and $50 \mathrm{~ms}$ and operating temperatures of (a) 20 , (b) 15 , and (c) $10^{\circ} \mathrm{C}$. The $R I$ is calculated before an initial two-point temperature correction process on the IRXCAM-160A camera.

and stable $1 / f$ noise under a specific electronic spectrum. It is interesting to observe that $R I$ decreases with a smaller deviation of the interpixel response on the arrays due to longer integration times and lower operating temperatures. The $R I$ can approach a value of $12 \times 10^{-3}$ (dimensionless). Simultaneously, the variations in $R I$ are stable even under the longer interval cycle of 300 frames. This means the real-time $R I$ monitoring becomes the essential factor for evaluating the performance of a thermal camera over a long operating period. Once the interval $R I$ value is below the threshold quantity, the recalibrated process for non-uniformity on the FPA may need to be implemented immediately to ensure high-performance thermal imaging.

Figure 7 shows the $R I$ as the function of the operating temperatures for "short" $(16.67 \mathrm{~ms})$ and "long" (50 ms) integration times before two-point temperature correction. We observe that, below $15{ }^{\circ} \mathrm{C}$, the differential values of the $R I$ between 16.67 and $50 \mathrm{~ms}$ are almost the same. For operating temperatures greater than $15^{\circ} \mathrm{C}$, the differential values are smaller than those for the temperature range of $10-15{ }^{\circ} \mathrm{C}$. This result indicates a larger degradation of the image (lower 


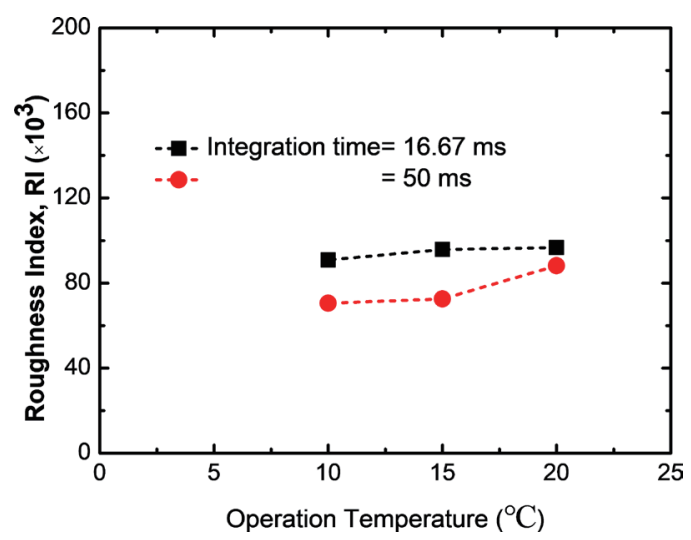

Fig. 7. (Color online) $R I$ as a function of operating temperatures $\left(10,15\right.$, and $\left.20^{\circ} \mathrm{C}\right)$ for short $(16.67 \mathrm{~ms})$ and long $(50$ $\mathrm{ms})$ integration times before two-point temperature correction.

$R I)$ with higher operating temperatures; that is, the $R I$ is more sensitive to higher integration times in the given range of operating temperature. We can deduce that a smaller difference between the operating temperature and blackbody temperature results in a larger variation in the signal response of the interpixels due to susceptibility to noise interference. In addition, we have derived the relations of RI between mean NETD and sigma NETD based on the Gaussian function $\left[f(x)=1 / \exp \left(\frac{x}{\sqrt{2} \sigma}\right)^{2}\right]$, where $\sigma$ is standard deviation. The FWHM is given by the distance between points on the curve at which the function reaches half its maximum value [FWHM $=2 \cdot \sqrt{2 \cdot \ln 2} \cdot \sigma]$. Applying the Gaussian function, the expression is revised as

$$
\operatorname{Counts}(N E T D)=a / \exp \left(\frac{N E T D-t}{\sqrt{2} \sigma}\right)^{2}
$$

where $a$ and $t$ are fitting parameters. The NETD values of all pixels (counts) in the microbolometer FPA are curve-fitted well under $T E C=20,15$ and, $10^{\circ} \mathrm{C}$ and frame rates of 10 , 15 and $30 \mathrm{~Hz}$, respectively. Where normally assumed $t \approx$ mean NETD and under the previous definitions and derivations, FWHM of NETD distribution is expressed as sigma NETD and its value is the same $2 \cdot \sqrt{2 \cdot \ln 2} \cdot \sigma$. In addition, $t / g=2 \cdot \sqrt{2 \cdot \ln 2} \cdot \sigma$, where $g$ is an experimental constant; we combine the two equations, then the mean NETD $\approx t=\operatorname{sigma} N E T D \cdot g$, where $g$ $\approx 2.09-2.23$. From Table 3, the ratio of sigma NETD and $R I$ equals approximately $2.62 \times 10^{-4}$, i.e., $R I \approx 3.8 \times 10^{-4} \cdot$ sigma $N E T D$. In summary, the relationship expressed with $R I \approx 3.8 \times 10^{-4}$ - sigma $N E T D \approx 1.76 \times 10^{-4} \cdot$ mean NETD is derived and behaves linearly.

Figure 8 shows photographs acquired with the thermal camera for the conditions described. Image (f) in Fig. 8 is of the highest quality (integration time of $50 \mathrm{~ms}$ and operating temperature of $10{ }^{\circ} \mathrm{C}$ with other conditions remaining constant). Images (d) to (f) in Fig. 8 (integration time of $50 \mathrm{~ms}$ ) exhibit higher contrast than those of images (a) to (c) (16.67 ms) under the same global electronic gain condition after the two-point temperature correction process. The tendency of the imaging results is consistent with the estimation of the $R I$ (Fig. 7). 


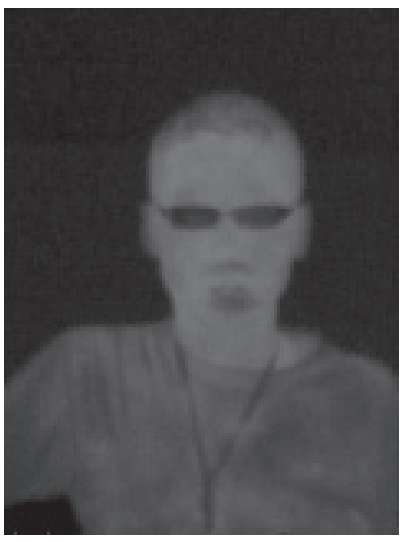

(a)

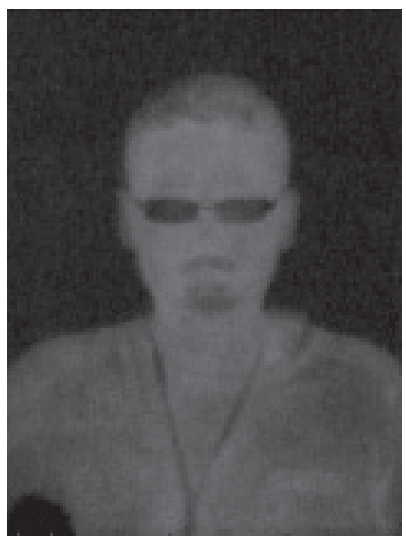

(d)

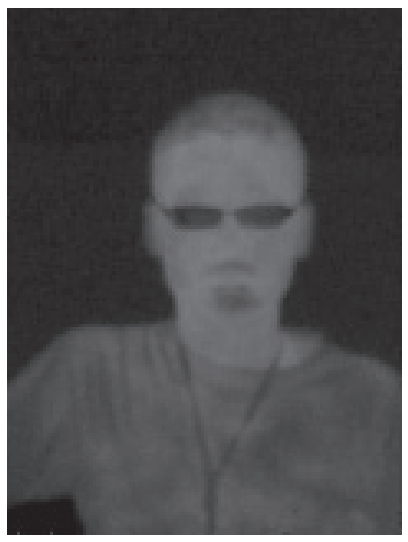

(b)

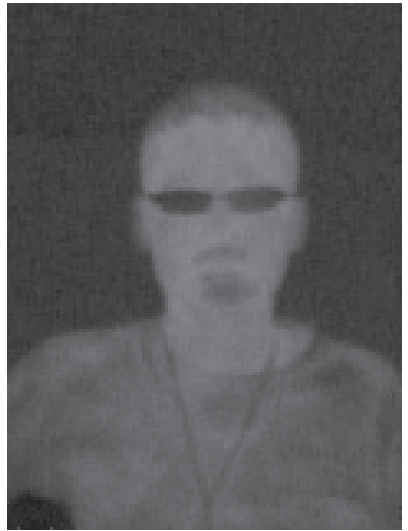

(e)

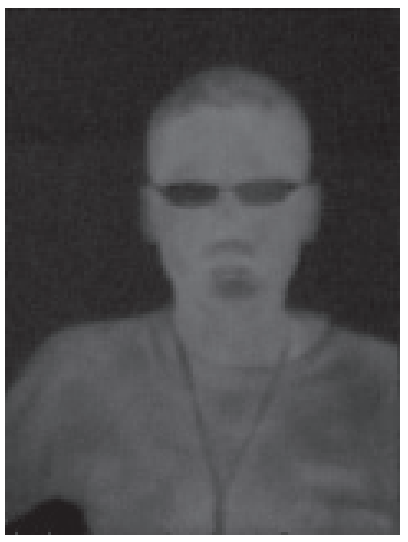

(c)

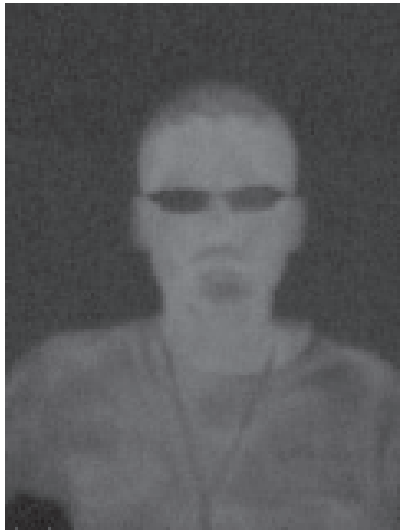

(f)

Fig. 8. Thermal photographs illustrating the dependence on integration times and operating temperatures: (a) $\tau=$ $16.67 \mathrm{~ms}, T_{s p}=20{ }^{\circ} \mathrm{C}$; (b) $\tau=16.67 \mathrm{~ms}, T_{s p}=15{ }^{\circ} \mathrm{C}$; (c) $\tau=16.67 \mathrm{~ms}, T_{s p}=10{ }^{\circ} \mathrm{C}$; (d) $\tau=50 \mathrm{~ms}, T_{s p}=20{ }^{\circ} \mathrm{C}$; (e) $\tau=$ $50 \mathrm{~ms}, T_{s p}=15^{\circ} \mathrm{C}$; and (f) $\tau=50 \mathrm{~ms}, T_{s p}=10^{\circ} \mathrm{C}$.

\section{Conclusions}

In this study, we evaluated the performance of a thermal imager based on the parameters of RI, NETD, and the FWHM of the NETD histogram. We determined that the imaging quality of the IR FPA is mainly degraded by temporal noise arising from higher operating temperatures for shorter integration times. Parameters of mean NETD and sigma NETD were mainly used for the evaluation of the thermal imaging camera, the parameters of which are closely related to the $R I$. From our results, we conclude that, via real-time monitoring, the $R I$ can be recalibrated to ensure better uniformity of the IR bolometer FPA when the level of the $R I$ is below a threshold value. Comprehensive parameters such as the mean NETD, sigma NETD, and RI are required for advanced uncooled VOx-bolometer-based thermal imagers for imaging predictions regardless of the integration time and operating temperature. We believe that our findings significantly contribute to the field of IR imaging. 


\section{Acknowledgments}

This work was supported by the Chung Cheng Institute of Technology (CCIT), National Defense University, National Chung-Shan Institute of Science and Technology (NCSIST), Department of Industrial Technology Ministry of Economic Affairs, and the Ministry of Science and Technology of the Republic of China under Contract No. NCSIST-310-V-404.

\section{References}

1 E. R. Deutsch, P. Kotidis, N. Zhu, A. K. Goyal, J. Ye, A. Mazurenko, M. Norman, K. Zafiriou, M Baier, and R. Connors: Proc. SPIE 9106 (SPIE, Maryland, 2014) 529-551.

2 A. Rogalski: Infrared Phys. Technol. 54 (2011) 136-154.

3 J. Lopez-Alonso: Encyclopedia of Optical Engineering, Vol. 2 (Marcel Dekker Inc., New York, 2003).

4 Z. Y. Wu, J. Y. Tsai, S. F. Tang, T. C. Chen, W. J. Lin, P. K. Weng, F. Picard, H. Oulachgar, T. Pope, and S. Ilias: Optomechatronic Technologies (ISOT), International Symposium (IEEE, 2014) 115-118.

5 D. Rafol, S. Gunapala, S. Bandara, and K. K. Law: Infrared Phys. Technol. 52 (2009) 371.

6 F. Niklaus, A. Decharat, C. Jansson, and G. Stemme: Infrared Phys. Technol. 51 (2008) 168.

7 M. J. Burke and W. H. Wan: Proc. SPIE 3377 (SPIE, Orlando, 1998) 71-76.

8 M. Hayat, S. Torres, E. Armstrong, S. Cain, and B. Yasuda: Appl. Opt. 38 (1999) 776.

9 S. Torres, R. Reeves, and M. Hayat: Proc. 6th World Multiconference on Systemics, Cybernetics and Informatics (International Institute of Informatics and Systemics, Albuquerque, 2002) 130-139.

10 J. Moreno, R. Redlich, G. Carvajal, and M. Figueroa: Programmable Logic (SPL), VIII Southern Conference (IEEE, 2012) 2.

11 A. Bergeron, H. Jerominek, C. Chevalier, L. L. Noc, B. Tremblay, C. Alain, A. Martel, N. Blanchard, M. Morissette, L. Mercier, L. Gagnon, P. Couture, N. Desnoyers, M. Demers, F. Lamontagne, F. Lévesque, S. Verreault, F. Duchesne, J. Lambert, M. Girard, M. Savard, and F. Châteauneuf: Proc. 3rd Int. Photonics \& OptoElectronics Meetings 276 (POEM, 2011) 012114.

12 W. Gross, T. Hierl, and M. Schulz: Opt. Eng. 38 (1999) 862.

13 M. Kohin and N. Butler: Proc. SPIE 5406 (SPIE, Orlando, 2004) 447-453.

14 S. I. Haider, S. Majzoub, M. Alturaigi, and M. Abdel-Rahman: IEIE Trans. Smart Process. Comput. 4 (2015) 251.

15 P. G. Datskos and N. V. Lavrik: Encyclopedia of Optical Engineering, Vol. 2 (Marcel Dekker Inc., New York, 2003).

\section{About the Authors}

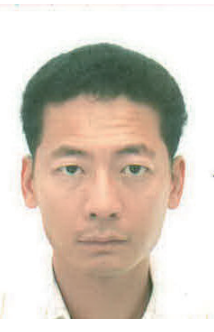

Cheng-De Lee received a BS degree from the Naval Academy, Kaohsiung, Taiwan and an MS degree in electronic engineering from the Chung Cheng Institute of Technology, Taoyuan, Taiwan, in 2000 and 2007, respectively. He is currently pursuing his Ph.D. at the School of Defense Science, Chung Cheng Institute of Technology, National Defense University. His research interests include signal and image processing and developing algorithms for infrared imagers. 


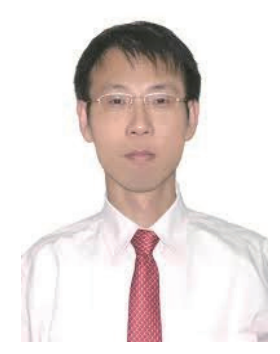

Shiang-Feng Tang received a BS degree in electrical engineering from the Chung Cheng Institute of Technology, Taiwan, in 1991, and MS and Ph.D. degrees in electrical engineering in 1996 and 2001, respectively, from the National Taiwan University, Taipei. He is currently an associate professor in the Department of Electrical and Electronic Engineering at the National Defense University, Taoyuan, Taiwan. His research interests include studying device design, fabrication, and evaluation of thermal imaging cameras based on the VOx focal plane array.

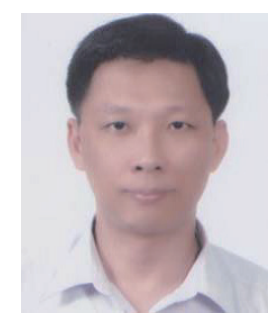

Tzu-Chiang Chen received his BS degree from the Chung Cheng Institute of Technology, Taoyuan, Taiwan, in 1988, and his MS and Ph.D. degrees in space science and optical science from the National Central University, Jhongli, Taiwan, in 1992 and 2000, respectively. He is currently a professor in the Department of Electrical and Electronic Engineering at the National Defense University, Taoyuan, Taiwan. His research interests include optical sensors and the modeling and fabrication of novel quantum optoelectronic devices. 